COCHRANE CORNER

${ }^{\dagger}$ This review is the abstract of a Cochrane review previously published in the Cochrane Database of Systematic Reviews, 2019, Mar 25 Issue 3: CD013069 (doi: 10.1002/ 14651858.CD013069.pub2) (see www.cochranelibrary.com for information). Cochrane reviews are regularly updated as new evidence emerges and in response to feedback, and the Cochrane Database of Systematic Reviews should be consulted for the most recent version of the review.

(C) 2020 The Cochrane Collaboration. Published by John Wiley \& Sons Ltd.

We thank the Cochrane Dementia and Cognitive Improvement Group for their support in publishing this review.

See commentary in this issue.

\section{Cognitive training for people with mild to moderate dementia: a Cochrane Review ${ }^{\dagger}$} Alex Bahar-Fuchs, Anthony Martyr, Anita M.Y. Goh, Julieta Sabates \& Linda Clare

\section{Background}

Cognitive impairment, a defining feature of dementia, plays an important role in the compromised functional independence that characterises the condition. Cognitive training is an approach that uses guided practice on structured tasks with the direct aim of improving or maintaining cognitive abilities.

\section{Objectives}

- To assess effects of cognitive training on cognitive and noncognitive outcomes for people with mild to moderate dementia and on their caregivers.

- To compare effects of cognitive training with those of other non-pharmacological interventions, including cognitive stimulation or rehabilitation, for people with mild to moderate dementia and their caregivers.

- To identify and explore factors related to intervention and trial design that may be associated with the efficacy of cognitive training for people with mild to moderate dementia and their caregivers.

\section{Search methods}

We searched ALOIS, the Cochrane Dementia and Cognitive Improvement Group Specialised Register, on 5 July 2018. ALOIS contains records of clinical trials identified through monthly searches of several major healthcare databases and numerous trial registries and grey literature sources. In addition to this, we searched MEDLINE, Embase, PsycINFO, CINAHL, LILACS, Web of Science Core Collection, ClinicalTrials.gov and the World Health Organization's trials portal ICTRP, to ensure that searches were comprehensive and up to date.

\section{Selection criteria}

We included randomised controlled trials (RCTs) that described interventions for people with mild to moderate dementia and compared cognitive training with a control or alternative intervention.

Data collection and analysis

We extracted relevant data from published manuscripts and through contact with trial authors if required. We assessed risk of bias using the Cochrane 'risk of bias' tool. We divided comparison conditions into active or passive control conditions and alternative treatments. We used a large number of measures and data to evaluate 19 outcomes at end of treatment, as well as 16 outcomes at follow-up in the medium term; we pooled this information in meta-analyses. We calculated pooled estimates of treatment effect using a random-effects model, and we estimated statistical heterogeneity using a standard $\chi^{2}$ statistic. We graded the evidence using GradePro.

\section{Main results}

The 33 included trials were published between 1988 and 2018 and were conducted in 12 countries; most were unregistered, parallel-group, single-site RCTs, with samples ranging from 12 to 653 participants. Interventions were between 2 and 104 weeks long. We classified most experimental interventions as 'straight cognitive training', but we classified some as 'augmented cognitive training', and about two-thirds as multidomain interventions. Researchers investigated 18 passive and 13 active control conditions, along with 15 alternative treatment conditions, including occupational therapy, mindfulness, reminiscence therapy and others.

The methodological quality of studies varied, but we rated nearly all studies as having high or unclear risk of selection bias due to lack of allocation concealment, and high or unclear risk of performance bias due to lack of masking (blinding) of participants and personnel.

We used data from 32 studies in the meta-analysis of at least one outcome. Relative to a control condition, we found moderatequality evidence showing a small to moderate effect of cognitive training on our first primary outcome, composite measure of global cognition at end of treatment (standardised mean difference $S M D=0.42,95 \% \mathrm{Cl} 0.23-0.62)$, and high-quality evidence showing a moderate effect on the secondary outcome of verbal semantic fluency (SMD $=0.52,95 \% \mathrm{Cl} 0.23-0.81$ ) at end of treatment, with these gains retained in the medium term (3-12 months posttreatment). In relation to many other outcomes, including our second primary outcome of clinical disease severity in the medium term, the quality of evidence was very low, so we were unable to determine whether cognitive training was associated with any meaningful gains.

When compared with an alternative treatment, we found that cognitive training may have little to no effect on our first primary outcome of global cognition at end of treatment $(S M D=0.21$, $95 \% \mathrm{Cl}-0.23$ to 0.64 ), but the quality of evidence was low. No evidence was available to assess our second primary outcome of clinical disease severity in the medium term. We found moderate-quality evidence showing that cognitive training was associated with improved mood of the caregiver at end of treatment, but this was based on a single trial. The quality of evidence in relation to many other outcomes at end of treatment and in the medium term was too low for us to determine whether cognitive training was associated with any gains, but we are moderately confident that cognitive training did not lead to any gains in mood, behavioural and psychological symptoms, or capacity to perform activities of daily living.

\section{Authors' conclusions}

Relative to a control intervention, but not to a variety of alternative treatments, cognitive training is probably associated with small to moderate positive effects on global cognition and verbal semantic fluency at end of treatment, and these benefits appear to be maintained in the medium term. Our certainty in relation to many of these findings is low or very low. Future studies should take stronger measures to mitigate well-established risks of bias and should provide long-term follow-up to improve our understanding of the extent to which observed gains are retained. Future trials should also focus on direct comparison of cognitive training with alternative treatments rather than passive or active control conditions. 